\title{
Beiträge zur Kenntnis des psychogalvanischen Phänomens.
}

\author{
Von \\ Privatdozent Dr. Adalbert Gregor, \\ II. Arzt der Klinik. \\ (Aus der psychiatrischen und Nervenklinik der Universität Leipzig \\ [Geh. Rat Paul Flechsig].)
}

(Eingegangen am 22. Dezember 1911.)

Das Studium der körperlichen Außerungen psychischer Zustände ist sowohl für die Psychologie wie für die Psychopathologie von Bedeutung, da es darauf ausgeht, an Stelle subjektiver Beobachtung objektive Werte zu setzen. Einen objektiven Maßstab für psychische Zustände zu gewinnen, erscheint besonders darum erwünscht, weil die subjektive, von der Selbstbeobachtung ausgehende Richtung schon im Bereiche der normalen Psychologie bewußter und unbewußter Täuschung ausgesetzt ist; die Psychopathologie aber zudem mit dem Mangel jeglicher Selbstbeobachtung zu rechnen hat. Daraus erklärt es sich, daß die experimentelle Psychologie besonders auf die Ausgestaltung und Vervollkommnung der zum Studium der Ausdrucksbewegung dienenden Methoden bedacht war, welche sie z. T. der Physiologie und Klinik entlehnte.

Der Stand der hier berührten Fragen wurde von mir anderen Orts unter dem Gesichtspunkte der Psychopathologie ${ }^{1}$ ) eingehend besprochen. Veranlassung zu dieser neuen Behandlung des Themas geben mir Studien, die sich in gleicher Richtung bewegten, aber mit einer neuen und aussichtsreichen Methode durchgeführt wurden. Es handelte sich dabei um die exakte Registrierung der an sich bereits länger bekannten, aber erst in jüngster Zeit genauer beachteten hautelektrischen Erscheinungen.

Auf die Geschichte der Methode ihrer Untersuchung brauche ich hier nicht näher einzugehen, da sie erst in letzter Zeit von verschiedenen Seiten $^{2}$ ) ausführlicher behandelt wurde. Ich begnüge mich daher mit der Bemerkung, daß zum Studium der hautelektrischen Erscheinungen

1) Gregor, Leitfaden der experimentellen Psychopathologie. Berlin 1910.

2) Albrecht, O., Experimentelle Untersuchungen über die Grundlagen der sogenannten galvanischen Hautelektrizität. Monatsschr. f. Psych. u. Neurol. 2y, 365, 439, 552. 1910. - Radecki, M. W., Recherches experimentales sur les phénomènes psycho-électriques. Arch. de Psychol. 11, 209. 1911. 
insbesondere zwei Apparate benützt werden, nämlich das Spiegel- und das Saitengalvanometer. Ersteres wird gewöhnlich in der sog. Veraguthschen Anordnung verwendet, welche darin besteht, daß der menschliche Körper in einen Stromkreis eingeschaltet wird. Dabei können bei gewissen psychischen Prozessen am Galvanometer Ausschläge beobachtet werden. Das Spiegelgalvanometer stellt aber, wie So $m \mathrm{mer}^{1}$ ) bemerkte, angesichts der feinen Potentialdifferenzen, die an der menschlichen Haut meßbar sind, ein schwer zu handhabendes Instrument vor, das wir heute durch ein bedeutend empfindlicheres, nämlich das Saitengalvanometer nach Einthoven ersetzen können. Dieses bietet in seiner von Edelmann hergestellten Ausstattung den weiteren großen Vorteil, daß es eine genaue photographische Registrierung der Saitenausschläge sowie der zeitlichen Verhältnisse gestattet. Die bisherigen Untersuchungen mit dem Spiegelgalvanometer zeigen nämlich meist den Mangel einer objektiven Registrierung der Galvanometerausschläge.

Bei der Beschreibung der Verwendung des Saitengalvanometers für unsere Zwecke gehe ich von der bekannten Versuchsanordnung bei der Aufnahme des Elektrokardiogrammes aus. Die Einschaltung der in Zinkwannen mit physiologischer Kochsalzlösung liegenden Extremitäten an die Galvanometersaite ergibt stets eine Ablenkung derselben. Wir müssen danach annehmen, daß unter diesen Bedingungen zwischen den beiden Metallwannen Potentialdifferenzen entstehen, welche das Auftreten des sog. Nullstromes bedingen. Dieser wechselt in seiner Stärke von Individuum zu Individuum und ist auch bei demselben Menschen nicht konstant Vor Aufnahme des Elektrokardiogrammes wird bekanntlich der Nullstrom durch Einschaltung eines entgegengesetzt gerichteten Stromes kompensiert; doch bleibt es keineswegs bei einer einmaligen Kompensation, vielmehr muß mitunter im Laufe einer längeren Herzaufnahme die Stärke dieses Stromes geändert werden. Ein deutliches Abweichen von dem Nullpunkt, um den die Saite bei dor Kardiographie schwingt, ist insbesondere auch dann zu beobachten, wenn man auf das Individuum, dessen Herzströme registriert werden, stärkere sensorische Reize ausübt, oder es veranlaßt, sich reproduktiv in eine lebhaftere Affektlage zu versetzen.

Versuche dieser Art wurden meines Wissens zum erstenmal von mir angestellt, um den Einfluß differenter psychischer Zustände auf die Herzaktion zu studieren. Ein solcher ist ja nach der Selbstbeobachtung ohne weiteres vorauszusetzen. Seine richtige Einschätzung fällt aber selbst bei kompliziertem instrumentellen Aufwande schwer, da die

i) Sommer, R., Die psychologischen Untersuchungsmethoden. Klinik für psychische $u$. nervöse Krankheiten 6, 205. 1911. 
Registrierung der Herzaktion mittels des Kardiographen infolge der starren und in seiner Lage veränderlichen Scheidewand (Brustkorb) ungenau ist und die mittels des Sphygmographen und Plethysmographen aufgenommenen Kurven keine sichere Entscheidung darüber zulassen, welche Änderung auf Rechnung des Herzens, welche auf die der Gefäße zu setzen ist. Meine Versuche ergaben, abgesehen von Modifikationen in der Frequenz des Herzschlages und Änderungen des Nullstromes, die wir später in geeigneterer Weise untersuchen wollen, ein negatives Resultat. Namentlich waren Änderungen in den Zacken der Elektrokardiographenkurven unter Einfluß der zum Studium der Ausdruckserscheinungen gewöhnlich verwendeten Reize, sowie bei reproduktiv ausgelösten Affekten zu vermissen. Der negative Ausfall dieser Versuche läßt aber doch keine Entscheidung darüber zu, ob unter den genannten Verhältnissen nicht doch auch Änderungen in der Stärke der Herzaktion auftreten, wie sie nach dem subjektiven Eindruck einerseits, sphygmound plethysmographischen Versuchen andererseits zu erwarten wären; denn spezielle Untersuchungen haben ergeben, daß ein strenger Parallelismus zwischen der Größe der Potentialdifferenzen bei der Herzaktion und der Kraft des Herzschlages nicht besteht.

Die zweite Möglichkeit, mittels des Saitengalvanometers hautelektrische Phänomene zu untersuchen, besteht darin, daß man die schon erwähnten Änderungen des Nullstromes genauer verfolgt. Wir sehen solche außer in der genannten Versuchsanordnung auch dann auftreten, wenn Metallelektroden, die die Versuchsperson in den Händen hält oder die an irgendeiner Körperstelle angelegt sind, an die Galvanometersaite angeschlossen werden. Ein Mittel, den Nullstrom erheblich zu steigern, besitzen wir in der Anwendung differenter Elektroden. Wie die grundlegenden Untersuchungen Sommers und seiner Schule lehrten, entstehen an der Berührungsstelle der menschlichen Haut mit verschiedenen Gliedern der galvanischen Spannungsreihe elektrische Ströme. So mmer und Fürsten a $\mathbf{u}^{1}$ ) konnten auch der menschlichen Haut eine bestimmte Stelle innerhalb der elektrischen Spannungsreihe und zwar zwischen Kupfer und Antimon zuweisen. Danach kann die Intensität des Nullstromes wesentlich erhöht werden, wenn als Elektroden Körper verwendet werden, die von der Stelle, welche die Haut in der galvanischen Spannungsreihe einnimmt, weiter entfernt sind. Dies ist z. B. dann der Fall, wenn wir an eine Körperstelle eine Zink-, an eine andere eine Kohlenelektrode anlegen. Wir haben es dann mit zwei hintereinander geschalteten Elementen Haut-Zink und HautKohle zu tun. Von Bedeutung für die Stärke der sich entwickelnden Ströme ist die Hautstelle, an die wir die Elektroden bringen und der

1) Sommer und Fürstenau, Die elektrischen Vorgänge an der menschlichen Haut. Klinik für psychische u. nervöse Krankheiten 1, 197. 1907. 
Zustand der Haut. Die stärksten Ströme sind bei der Wahl schweißreicher Körperstellen zu beobachten (Handteller, Fingerspitzen, Fußsohlen). Intensive Behandlung mit Alkohol, Beizung mit Formalin reduziert sie wesentlich. Von Einfluß auf die Stromstärke ist auch die Größe der Kontaktfläche und die Stärke des Druckes, unter dem die Elektrode anliegt. Ein genaueres Studium dieser Momente gestatten die von Sommer nach Art von Briefwagen konstruierten Elektroden, welche mit durchlöcherten Kautschukmembranen überzogen werden.

Ich begnüge mich mit diesen Bemerkungen über die Hautströme; ihre Kenntnis erscheint notwendig, da die erwähnten Faktoren als Fehlerquelle bei jeder Untersuchung in Betracht kommen. Ein näheres Eingehen auf die physikalische Seite der Erscheinung, die in jüngster Zeit von verschiedenen Autoren studiert wurde, erübrigt sich hier. Da diese Untersuchungen noch $\mathrm{z}$. T. widersprechende Resultate ergaben, wurde dieser Seite der Frage in unserem Laboratorium eine besondere Untersuchung gewidmet. Ehe Klärung erfolgt, können die unter verschiedenen Versuchsanordnungen gemachten Beobachtungen nicht identifiziert werden, nur die oft unverkennbare Ubereinstimmung ihrer Resultate berechtigt uns, die verschiedenen Untersuchungen in Parallele zu stellen.

Wie über die physikalische und physiologische Natur der hautelektrischen Erscheinungen gehen die Ansichten der Autoren auch über die Art der psychischen Prozesse auseinander, welche vom psychogalvanischen Phänomen begleitet werden. Nach Tarchanoff ${ }^{1}$ ), Veraguth${ }^{2}$ ) und $S \operatorname{Stach}^{3}$ ) ist das psychogalvanische Phänomen nicht an besondere Bewußtseinserscheinungen gebunden. Nach Sidis und Kalmus s $^{4}$ begleitet es lebhafte Empfindungen und Gefühle, nicht aber intellektuelle Vorgänge. Demgegenüber sucht Radecki zu beweisen, daß psychogalvanische Erscheinungen nur bei affektiven Erregungen auftreten und daß jede affektive Erregung eine solche Reaktion erzeugt. Radecki, der die Frage eingehender studierte, hat als Stütze seiner Ansicht gewichtige Gründe angeführt. Bei der großen Tragweite dieser Behauptung speziell für die Psychopathologie wäre es angezeigt, seine mit der Veraguthschen Anordnung getroffenen Versuche einer Nachprüfung mit dem Saitengalvanometer zu unter-

1) Tarchanoff, v., Über die galvanischen Erscheinungen an der Haut des Menschen bei Reizungen der Sinnesorgane und bei verschiedenen Formen der psychischen Tätigkeit. Archiv f. d. ges. Physiol. 46, 46. 1890.

2) Veraguth, O., Das psychogalvanische Reflexphänomen. Monatsschr. f. Psych. u. Neurol. 21, 387. 1907.

3) Starch, D., Mental Processes and concomitant galvanometric Changes. Psychol. Review 17, 19. 1910.

4) Sidis and Kalmus, A Study of galvanometric Deflections due to Psychophysical Processes. Psychol. Review 15, 390. 1908. 16, 1. 1909. 
ziehen. Nach den erwähnten Faktoren, welche auf das Zustandekommen, namentlich aber auf die Intensität der Reaktion von Einfluß sind, werden individuelle Unterschiede in der Reaktionsweise nur bei Berücksichtigung besonderer Kautelen in der äußeren Versuchsanordnung, wie konstanter Druck und konstante Berührungsfläche der Elektroden und gleiche Vorbehandlung der Haut ermittelt werden können. Noch schwieriger gestaltet sich die Aufgabe, von konstanten inneren Bedingungen auszugehen. Die bisher in dieser Richtung ausgeführten Untersuchungen haben die genannten Fehlerquellen zu wenig berücksichtigt, als daß wir ihnen vollen Wert zuerkennen könnten. Radecki fand mit dem Spiegelgalvanometer bei sensiblen Jndividuen oft auffallend schwache Reaktionen und erklärt diese Erscheinung durch Interferrenz verschiedenfältiger Erregungen, die von den Reizen ausgingen. Die von $\mathrm{ihm}$ gefundenen individuellen Differenzen in der Größe der Ausschläge, in der Dauer der Latenzzeit und in der Anspruchsfähigkeit auf Reize waren zu variabel, als daß an eine Einteilung der Versuchspersonen nach ihrer Reaktionsart gedacht werden konnte. Er mußte sich daher auf den Versuch beschränken, feste Gruppen von Individuen nach ihrer Reaktionsweise zu charakterisieren. Frauen zeigten große Galvanometerausschläge während der Erwartung des Reizes, Männer auf den Reiz selbst. Dies Ergebnis zeigt, daß die ganzen, an einer großen Zahl von Individuen durchgeführten Versuche ihren Zweck verfehlten. Infolge der ungleichen inneren Versuchsbedingungen sind dabei nicht die Reaktionen vergleichbar, sondern bloß der Zustand vor Ausübung des Reizes. Die durch den Reiz selbst ausgelösten Reaktionen sind ohne genaueste Analyse des psychischen Zustandes, der vor und nach Applikation des Reizes bestand, nur von beschränktem Werte; und eine solche Analyse ist bei Radecki zu vermissen. Meine an normalen und pathologischen Individuen angestellten Versuche ließen mich bald erkennen, daß derartige, schon ins Gəbiet der Mental Tests reichenden Versuche wie bisher auf keinem Gebiete der Psychologie auch beim Studium des psychogalvanischen Phänomens nicht zulässig sind. Selbst bei normalen und intelligenten Versuchspersonen bedarf es hier einer erst durch zahlreiche Versuche zu gewinnenden Gewöhnung an die Versuchsbedingungen, um Reaktionen zu bekommen, bei denen von einer Vergleichbarkeit die Rede sein kann. Bei aller Instruktion zeigen selbst normale und in anderweitigen Versuchen geübte Individuen unregelmäßige, kaum zu deutende Schwankungen; erst im Laufe der Zeit tritt beim Experimente eine gleichmäßige, indifferente Gemütsstimmung ein, die als Ausgang vergleichbarer Versuche dienen kann. Dieser Zustand findet auch objektiv in den mit dem Saitengalvanometer gewonnenen Kurven in einer besonderen Ruhekurve seinen Ausdruck. Wie später noch gezeigt 
werden wird, schließen sich Zustände, wie sie Radecki bei Personen mit deutlicher Erwartungskurve vor sich hatte und ausgiebige Reaktionen auf einfache Reize gegenseitig aus.

Nach dem Gesagten eignet sich die psychogalvanische Methode von vornherein keineswegs zur Untersuchung flüchtiger psychischer Veränderungen. Derartige Zustände werden ihr nur unter besonders günstigen Bedingungen, die an Zufall streifen, zugänglich werden. Wie vorauszusehen, hatten wir mit den besprochenen Schwierigkeiten auf psychopathologischem Gebiete in erhöhtem Maße zu kämpfen. Ein großer Teil von Patienten ist als Versuchsperson für derartige Untersuchungen ungeeignet, weil motorische Erregungs- oder Reizzustände, inkonstante Gemütslage, Ängstlichkeit, Ablenkbarkeit usw. keine geeignete Ruhekurve, die als Basis von vergleichenden Versuchen dienen konnte, zustande kommen ließen. Eine systematische Untersuchung muß ebensowohl mit individuellen Faktoren wie mit dem vorliegenden Krankheitsbilde rechnen. Bei den genannten Schwierigkeiten in der Untersuchung von Geisteskranken können wir die von Cla parèd $\mathrm{e}^{\mathrm{l}}$ ) gemachten Beobachtungen nur mit Reserve aufnehmen. Seine daraus gezogenen Schlüsse werden wir aber jetzt schon entschieden ablehnen müssen. Cla parède untersuchte die psychogalvanische Erregbarkeit an 4 hochgradigen Idioten und vermißte bei ihnen die geringste Spur einer Reaktion, woraus er schloß, daß das Zustandekommen derselben Intaktheit der Hirnrinde zur Voraussetzung habe.

Aus meinem bisher gesammelten Materiale von Beobachtungen über das psychogalvanische Phänomen möchte ich zunächst die bei den zahlreichen Versuchen gewonnenen Kurven ihrer Form nach einer genaueren Besprechung unterziehen. Denn, wie bereits bemerkt, muß die Analyse des Charakters einer Kurve der Bewertung der einzelnen Reaktionen vorausgehen. Ich verfüge bisher über 80 Kurvenfolgen, die in ebensoviel Sitzungen gewonnen wurden. Jede Kurvenfolge enthält eine Reihe von Kurven, die den Reaktionen einer Versuchsperson in einer Sitzung entsprechen. Als Versuchsperson verwendete ich bisher Normale, Neurastheniker, konstitutionelle und traumatische Hysteriker, Fälle mit organischen Nervenkrankheiten, Melancholiker, Katatoniker, Hebephrene, Paralytiker. An der Mehrzahl dieser Versuchspersonen wurde eine Reihe von einfachen Versuchen in verschiedenen zeitlichen Abständen hintereinander ausgeführt. (Einfacher akustischer Reiz [Glocke], Pinselberührung, angenehmer Geruch, Vorzeigen eines Bildes, unangenehmer Geruch, Vorzeigen eines gedruckten Wortes, Stich, Zählen von Punkten, einfache Rechenaufgabe.) Geeignete Versuchspersonen wurden zu weiteren, später zu

1) Cla parède, E., Société de physique et d'histoire naturelle de Genève. Extrait des Archives des Sciences physiques et naturelles. Avril 1911. 
erörternden Versuchen herangezogen. Rein deskriptiv vorgehend, kann ich die in den beschriebenen Versuchen an Normalen und Geisteskranken gewonnenen Kurven in mehrere Gruppen einteilen:

1. Kurven, die im Ruhezustand leicht wellenförmig verlaufen, dabei ganz sanft ansteigen. Die Reaktionen sind meist ausgiebig.

2. Kurven mit ausgesprochenen Wellenbergen und Wellentälern und stärkerem Anstiege. Die Galvanometerausschläge sind wechselnd, meist weniger groß als im ersten Falle.

3. Kurven von geradlinigem Verlauf, die horizontal sind oder nur wenig ansteigen. Die Reaktionen sind hier verschieden groß, fehlen mitunter ganz.

4. Kurven von geradlinigem Verlauf und deutlichem Anstieg. Diese zeigen öfters abrupte Schwankungen, die auf Muskelzuckungen oder Tremor zurückzuführen sind. Die Reaktionen sind klein oder fehlen ganz. Der absteigende Kurvenschenkel erreicht die Abszisse des Fußpunktes vom aufsteigenden nicht. Oft ist die Reaktion überhaupt nur durch einen stärkeren Anstieg markiert.

Bei der Deutung dieser Befunde gehen wir von dem uns sicherer zugänglichen Bewußtseinszustande des Normalen aus. Nimmt man, wie es nach dem jetzigen Stande der Frage am wahrscheinlichsten ist, den im Kurvenbilde als Welle erscheinenden Galvanometerausschlag als Ausdruck einer emotionellen Erregung an, so entspricht die Wellenfolge, wie wir sie in Gruppe 2 finden, einer Folge von affektiven Erregungen. Dazu stimmt die Beobachtung, daß wir derartige Kurven bei Normalen und Neurasthenikern fanden, welche unter Einfluß des Experimentes deutlich in Erregung gerieten. Ausgesprochene Kurven dieser Art beobachteten wir besonders auch bei Hysterischen, ferner bei einem Falle von Katatonie, der durch das Experiment in ängstliche Erregung versetzt wurde.

In dem Maße als sich die normalen Individuen an die Eigenart der Versuche gewöhnten und die Erregung zurücktrat, ging der Typus 2 in den Typus 1 über. Ich sah diesen aber auch in Versuchen mit Geisteskranken auftreten. Bei Normalen ist mit vollständiger Gewöhnung an die Versuchsbedingungen ein Utbergang dieser Kurvenform in den Typus $3 \mathrm{zu}$ beobachten. Kurven mit geradlinigem Verlaufe und leichtem oder fehlendem Anstieg fanden wir bei Normalen, die öfter zu Versuchen herangezogen wurden. Die Ausschläge waren meist ausgiebig. Kurven von gleichem Verlaufe fanden wir aber auch bei katatonem Stupor, motorischen Hemmungszuständen, bei Psychasthenie, Melancholie ohne Affekt und bei progressiver Paralyse. Die Reaktionen der Melancholiker waren klein und fehlten bei starker Hemmung fast ganz. Bei anregbaren Melancholikern wurden sie um so stärker, je mehr ihre psychische Leistungsfähigkeit in Anspruch genommen wurde (kom- 
pliziertere Rechenaufgaben usw.). Ein derartiger Fall zeigte dabei den stärksten Ausschlag, als er infolge eines Mißverständnisses sich vor eine für ihn unlösbare Aufgabe gestellt sah und darïber in Affekt geriet. In einem Falle von psychasthenischer Hemmung war der Utbergang der geradlinigen Kurvenform in den Typus $2 \mathrm{zu}$ bemerken, als im Versuche eine emotionelle Erregung erzeugt wurde. Wir sehen hier das Gegenteil von der beim Normalen mit zunehmender Beruhigung eintretenden Änderung der Kurvenform. Einen geradlinigen, horizontalen Kurvenverlauf fand ich bei katatonem Stupor, doch waren hier die Reaktionen klein. Ähnliche Kurven gewannen Jung und Peterson $\left.{ }^{1}\right)$ durch Registrierung der Ausschläge des Spiegelgalvanometers in ihren Versuchen an Katatonikern. Einzelne Individuen, die etwas freier sind, reagieren noch auf stärkere affektive Reize, wie Verheißung, nach Hause zu kommen. Aber schon etwas stärkere Stuporformen ließen jede Reaktion vermissen. So u. a. eine Frau meiner Beobachtung, die klinisch wohl deutlich gehemmt, aber nicht akinetisch war, vielfach spontane Bewegungen machte, selbst aß, sich ankleidete usw., auf der Abteilung gelegentlich Freude äußerte. Dabei ist zu bemerken, daß diese Patientin den Reizen ihre Aufmerksamkeit zuwendete, wie durch parallele Versuche mit Registrierung der Atmung mit Sicherheit zu entscheiden war. Diese wurden in ähnlicher Weise angestellt wie die seinerzeit veröffentlichten Versuche über die Diagnose psychischer Prozesse im Stupor ${ }^{2}$ ). Die Patientin lag in ruhiger Rückenlage im Bett, eine Atemkapsel wurde an ihre Brust, die beiden an das Galvanometer angeschlossenenen Elektroden an die Hände gelegt. Die ersten beiden Reize, Glocke und Stich, erzeugten keine Veränderung im Atemrhythmus, auf Ammoniak typische Verkürzung. Bei dem nun folgenden Reiz (Bild) wieder normales Atmen. Auf Zuruf ,nun kommt etwas Unangenehmes“ neuerliche Atemverkürzung; damit ist der Nachweis geliefert, daß die Patientin diesen Reiz aufgefaßt hatte. Die gleichzeitig aufgenommene psychogalvanische Kurve verlief während der ganzen Versuchsreihe geradlinig, ohne die Abszisse zu verlassen. Man könnte vielleicht geneigt sein, nach dem Ausfall dieser beiden parallelen Versuche Schlüsse auf die Schärfe der beiden, zur Registrierung der Ausdrucksbewegungen dienenden Methoden zu ziehen. Darum sei ausdrücklich bemerkt, daß die hier beobachteten Atemveränderungen keine emotiven Erregungen zur Voraussetzung haben müssen. Dagegen stellte ich vergleichende

1) Peterson, F. and C. G. Jung, Psychophysical Invastigations with the Galvanometer and Pneumograph in normal and Insane Individuals. Brain 30, 153. 1907.

2) Gregor, A., u. Zaloziecki, A., Diagnose psychischer Prozesse im Stupor. Klinik f. psychische u. nervöse Krankheiten 3, 22. 1908. 
Versuche bei mehreren Patienten in der Weise an, daß bei ihnen unter Anwendung gleicher Reize sphygmographische und psychogalvanische Aufnahmen erfolgten. Derartige Versuche fielen durchaus zugunsten der psychogalvanischen Methode aus, indem sie bei Versuchspersonen positive Resultate ergab, bei denen auf gleiche Reize keinerlei Veränderungen im Sphygmogramm nachzuweisen waren.

Endlich ist zu erwähnen, daß die uns hier beschäftigende Kurvenform (Typus 3) auch bei stumpfen Paralytikern nachzuweisen war. Hier traten weder auf einfache, noch auf affektive Wortreize irgendwelche Schwankungen im Verlaufe der Kurve auf. Nach der Auffassung der Galvanometerablenkungen als Ausdruck emotioneller Erregung würden derartige Kurven für einen Mangel an Anspruchsfähigkeit auf emotive Reize sprechen, wobei es jetzt noch unentschieden bleiben muß, ob es im besonderen Falle mehr an dem Ausdruck oder auch an dem subjektiven Zustande selbst fehlt. An die erste Möglichkeit wäre bei Katatonikern zu denken; bei Paralytikern kommt wohl eher die zweite in Betracht; unter bestimmten Bedingungen könnten solche Kurven auch jetzt schon diagnostische Bedeutung gewinnen.

Kurven vom Typus 4 (geradlinig mit erheblicher Steigung ohne deutliche Ausschläge) beobachtete ich bei normalen Individuen, die sich infolge der ganzen Anlage der Versuche oder infolge einer besonderen Aufgabe in starker Aufmerksamkeitsspannung befanden. Der affektive Zustand einer Versuchsperson, die Konzentration andererseits machen den geradlinigen Anstieg verständlich. Der Mangel der gewöhnlichen Reaktionen auf gleiche Reize ist dadurch zu erklären, daß neben dem primären Affekte keine neue emotive Erregung erfolgte. Eine gleiche Kurve fand ich bei einem aggravierenden Traumatiker, der allen klinischen Untersuchungen mit gespannter Aufmerksamkeit folgte und seine dabei gemachten Erfahrungen in den nächsten Untersuchungen verwertete. Ferner zeigten sie einzelne Fälle von Katatonie mit Angstsymptomen.

Die hier gegebene Úbersicht zeigt, daß wir übereinstimmende Kurven fanden, wenn bei normalen und geisteskranken Versuchspersonen qualitativ ähnliche Bewußtseinszustände vorlagen. Wir können jetzt schon gewissen affektiven Zuständen besondere Kurven oder Kurvenmerkmale zuweisen, und zwar dem indifferenten Gemütszustande eine geradlinige, wenig oder gar nicht ansteigende Kurve; der Apathie den Mangel an Galvanometerausschlägen bei Anwendung affektiver Reize; der Erregung einen wellenförmigen; der Spannung einen geradlinigen, stärker ansteigenden Kurvenverlauf mit unausgiebigen Reaktionen auf einfache Reize. Weitere Untersuchungen, die bereits im Gange sind, werden die Frage erörtern, ob die galvanometrischen Kurven noch eine feinere und speziellere. Charakteristik 
emotioneller Zustände geben können. Mit Rücksicht auf diese Arbeit verzichte ich darauf, die hier angegebenen Befunde mit der Reproduktion von Kurven zu belegen ${ }^{1}$ ).

Bis jetzt beschäftigten uns' die galvanometrischen Kurven mehr nach ihrem allgemeinen Charakter. Im folgenden wird es mitunter auf den Vergleich der einzelnen Reaktionen ankommen. Wie weit wir $\mathrm{zu}$ einem solchen überhaupt berechtigt sind, sei vorerst kurz erörtert. Technische Momente zwingen da, wo der absteigende Kurvenschenkel die Abszisse nicht erreicht, dies durch Änderung des kompensierenden Stromes zu veranlassen. Diese Änderung kann aber natürlich für die weiteren Reaktionen nicht gleichgültig sein. Aber auch wenn der Nullpunkt der Ordinate wieder erreicht wird, nehmen nach den bisherigen Beobachtungen aufeinanderfolgende Reaktionen im Laufe einer Sitzung an Größe ab. Zur Erklärung dieser Erscheinung können physikalische wie psychologische Faktoren angeführt werden. Von Interesse wäre jedenfalls die noch mangelnde Feststellung der entsprechenden zeitlichen Verhältnisse unter Berücksichtigung des Nullstromes. Es wäre also zu untersuchen, von welcher Dauer des Intervalles an bei qualitativ und quantitativ gleichen Reizen eine Abnahme der Reaktion erfolgt. Für uns kann zunächst die Einsicht genügen, daß aufeinanderfolgende Reaktionen bei demselben Individuum miteinander nicht ohne weiteres vergleichbar sind. Dagegen sind wir aber zweifellos berechtigt, eine Reaktion mit größerem Galvanometerausschlag für stärker als vorausgehende mit kleineren Ausschlägen anzusprechen.

Eine Frage von allgemeinerem ärztlichen Interesse betrifft die Ausdruckserscheinungen bei den verschiedenen Formen von Analgesie. Sie hat begreiflicherweise die auf diesem Gebiete tätigen Autoren seit langem beschäftigt, aber mit den früher verfügbaren Methoden (Sphygmo- und Plethysmographie) noch keine befriedigende Lösung gefunden. Veraguths ${ }^{2}$ ) Versuche an organischem Material (Nervenund Rückenmarkskranke, Lokalanästhesie) ergaben z. T. positive und vollkommen plausible Ergebnisse, nämlich Mangel einer galvanischen Reaktion bei Reizen, die nicht empfunden wurden und Parallelismus zwischen subjektiven Angaben über die Stärke der Empfindung und Galvanometerausschlag. Doch gab es auch negative Fälle, Reize wurden empfunden, aber es fehlte eine Reaktion, oder Empfindungen von verschiedener Stärke entsprachen gleiche Galvanometerausschläge. Nach dem bereits Gesagten kommen für die Deutung dieser Erscheinungen verschiedene Möglichkeiten in Betracht. Besondere Beachtung

1) Ein Teil des Materials wurde von mir bei der XVII. Versammlung mitteldeutscher Psychiater u. Neurologen am 22. Oktober 1911 demonstriert.

2) Veraguth, O., Das psychogalvanische Reflexphänomen. Monatsschr. f. Psych. u. Neurol. 23, 204. 1908. 
verdient ein Befund. Bei einem Tabiker mit verlangsamter Reaktion und langem Nachschmerz fand Verag uth deutliche Galvanometerausschläge, dabei wurde die Empfindung 2 Sekunden nach Applikation des Reizes gemeldet, die galvanische Reaktion trat erst nach weiteren 5 Sekunden auf. Veraguth geht auf den Fall nicht näher ein. Auffallend erscheint dabei die lange Latenzzeit und die im Verhältnis zur Perzeption stark verspätete Reaktion, die entschieden genauere Beachtung verdient. Einen bisher scheinbar noch nicht beobachteten Befund ergaben Versuche an einem Tabiker aus meinem Material. Klinisch lag deutlich Hypalgesie und verlangsamte Schmerzleitung bei Reizen an der Fußsohle vor. Ich fand bei Reizung der normal empfindenden Hand prompte galvanische Reaktion, bei Stich auf die Fußsohle trat nach 7 Sekunden eine positive und deutlich ausgesprochene Galvanometerablenkung auf. Dabei wurde aber keine Empfindung ausgelöst. Wir haben es hier also mit einer Reaktion auf einen nicht empfundenen Reiz zu tun, wobei die Reaktion im Sinne einer verlangsamten Schmerzleitung erfolgt. Die eine Seite dieser Reaktionsform, nämlich positiver Ausfall bei mangelnder Empfindung tritt häufig bei funktionellen Störungen auf. Damit berühren wir ein Gebiet von großem praktischen Interesse.

Funktionelle Anästhesien und Analgesien wurden von Veraguth ${ }^{\mathbf{1}}$ ) und Moravesik ${ }^{2}$ ) mit der psychogalvanischen Methode untersucht. Verag u th kam nach Versuchen an einer Reihe von hysterischen Anästhesien zu dem Ergebnis, daß in diesen Fällen bei völligem oder fast vollständigem Ausbleiben jeglicher subjektiven Äußerung oder bei strikter Versicherung der Versuchsperson, daß sie von kräftigsten Reizen nichts spürt, am Galvanometer starke Ausschläge auftreten und zwar im allgemeinen größere bei Reizen auf der analgetischen als auf der gesunden Seite. Veraguth denkt dabei an eine differentialdiagnostische Verwertung dieser Befunde gegenüber organischen Analgesien. Andere Befunde konnte Moravcsik erheben und kam seiner Ansicht nach zu einem von Verag uth abweichenden Resultate. Er suggerierte einem hysterischen Individuum vollkommene (taktile, akustische, optische Unempfindlichkeit) und fand keine oder geringe Ausschläge. Bei Besprechung eines Versuches, in dem bei suggerierter Taubheit das Galvanometer auf lebhafte akustische Eindrücke und Mitteilung einer der Versuchsperson angenehmen Nachricht keine Ausschläge zeigte, scheint der Autor einen gewissen Gegensatz zu der bekannten Tatsache zu empfinden, daß Hypnotisierte akustische Reize doch perzipieren, da sie ja mündlichen Befehlen nachkommen. Eigene Versuche in

1) Veraguth, l. c.

2) Moravcsik, E., Experimente über das psychogalvanische Reflexphänomen. Journ. f. Psychol. u. Neurol. 18, 186. 1911. 
dieser Richtung führte ich zunächst an einem sehr zuverlässigen Individuum aus, das in der Hypnose stark suggestibel ist. Bei Schmerzreizen auf die durch Suggestion analgetisch gemachte Hand bekam ich Kurven, die einer organischen Analgesie vollkommen glichen. Unter denselben Versuchsbedingungen fand ich bei. derselben Versuchsperson auch Reaktionen, die jenen der normal empfindenden Hand entsprachen, mitunter sogar lebhaftere Wellenbewegungen zeigten.

Die Untersuchung von hysterischen Patienten, bei denen halbseitige Analgesie ein bewußtes oder unbewußtes Symptom war, ergab ganz ähnliche Kurvenbilder. Ich fand auch hier bei denselben Individuen Fehlen oder minimale Reaktion auf der kranken Seite, abwechselnd mit deutlichen Reaktionen, welche denen der normalen Seite entsprachen oder sie an Größe der Ausschläge übertrafen.

Die Deutung der im obigen erwähnten eigenen und fremden Befunde fällt nicht schwer, wenn man auf dem Standpunkt steht, daß die hysterische sowie die posthypnotische Analgesie eine Ausschaltung oder Verdrängung von Empfindungen vorstellt und daß bei diesem Vorgange Komplexe beteiligt sind, die mindestens bei Hysterischen oft stark affektbetont sind. Ein Anklingen derselben kann natürlich zu lebhafteren Reaktionen führen. Andererseits hat es aber auch nichts Befremdliches, daß ein weniger affektbetonter Komplex, wie er z. B. vielfach bei hypnotischen Experimenten an leicht suggestibeln und vielfach hypnotisierten Individuen vorliegen dürfte, zu keinen stärkeren Reaktionen Anlaß gibt. Weniger empfindlich sind wohl auch alte, stereotypierte Komplexe, die wir bei Hysterikern finden, die der Demenz nahe stehen. Die Untersuchung derartiger Fälle, bei denen man klinisch, falls ein Trauma in Betracht kommt, leicht an Simulation denkt, wird denjenigen, der durch diese Methode Simulanten zu entlarven denkt, nicht befriedigen. Endlich ist das Auftreten von stärkeren Reaktionen hier mitunter schon durch den besonderen Bewußtseinszustand, Spannung, Erregung, ausgeschlossen. Als Stütze meiner Ansicht kann ich auch Beobachtungen anführen, die Radecki unter anderen Gesichtspunkten unternommen hat. Er versuchte den Einfluß des Willens auf die psychogalvanische Reaktion zu bestimmen. Dabei wurde unter anderem auch die Instruktion gegeben, durch Willensanstrengung die Reaktionen zu verhindern; einzelne Personen bekamen auch den Auftrag sich willkürlich ruhig zu verhalten. Die Versuche ergaben eine Verminderung der Reaktionen, doch brachte es keine Versuchsperson fertig, konstant gar nicht zu reagieren, im Gegenteil wurde zuweilen durch eine derartige Bemühung die Reaktion sogar gesteigert. Leider hält Radecki diese prinzipiell verschiedenen Versuche nicht genügend auseinander. Die Instruktion, sich ruhig zu 
verhalten, mußte doch wohl eine ganz andere psychische Verfassung der Versuchsperson veranlaßt haben, als der Auftrag, nicht zu reagieren. Letzterer ist wieder zu unbestimmt, um durchsichtige Versuche zu ermöglichen. Wir dürfen aber annehmen, daß die Versuchspersonen selbst auf den Gedanken kamen, sich dem Eindruck des Reizes zu verschließen und seine unmittelbaren psychischen Wirkungen aus ihrem Bewußtsein auszuschalten. Dadurch kamen sie aber hysterischen Individuen psychologisch nahe. Die Úbereinstimmung der Resultate in den galvanometrischen Aufnahmen kann infolgedessen nicht. überraschen. Exaktere Versuche, ähnlich wie sie Radecki anstellte, mit genauerer Beherrschung der Versuchsbedingungen würden gewiß noch weiter führen.

Nach unserer jetzigen Erfahrung stellt sich die differentaldiagnostische Seite der Frage weniger einfach dar, als es Veraguth erschien. Wir haben ja nun auch bei funktionellen Analgesien negative Reaktionen gefunden. Nach dem jetzigen Stande der Forschung ist zu sagen: ein Teil der Fälle unterscheidet sich durch deutliche Reaktionen, die eventuell über die normalen hinausgehen, von organisch Kranken, deren Reaktionen sich von normalen durch Kleinheit und verspätetes Auftreten deutlich abheben. Bei einer zweiten Gruppe sind Reaktionen ähnlich wie bei organisch Kranken zu vermissen. Nach.unseren jetzigen Erfahrungen können wir aber aus dieser Gruppe durch umfangreichere Versuche, die sich nicht nur auf das analgetische Gebiet und auf eine Empfindungsqualität beschränken, eine Charakteristik des Individuums zumal in seiner allgemeinen Reaktionsweise gewinnen. In manchen Fällen wird im Gegensatz zu organisch Kranken eine gleichmäßige Herabsetzung der psychogalvanischen Reaktion auf differente Reize zu beobachten sein. Ob dann noch eine Restgruppe verbleibt, die durch unsere Methode von organisch Kranken nicht differenzierbar ist, wird erst eine ausgedehntere spezielle Untersuchung eines größeren Materiales ergeben.

An die Unterscheidung zwischen organischen und funktionellen Analgesien schließt sich meist direkt die Frage, ob etwa aus Simulationstendenz oder infolge eines funktionellen Leidens eine .Verminderung der Reaktionen besteht. Wie bereits erwähnt, läßt das vorliegende Material eine sichere Entscheidung noch nicht zu. Von Bedeutung dürfte hier die Kurvenform werden. Geradlinige, horizontale oder leicht wellenförmige Kurven mit deutlichen Ausschlägen auf differente Reize aber fehlenden Reaktionen auf Reizung des angeblich analgetischen Gebietes, halte ich bei Simulation für ausgeschlossen. Verdacht auf Simulation werden wir hingegen bei den oben beschriebenen Spannungskurven schöpfen müssen. Solche sind zwar auch bei funktionellen Krankheiten zu beobachten, verlieren sich aber im Ver- 
laufe der Versuche, wobei gleichzeitig deutliche Ausschläge auf den Gebieten mit erhaltener Sensibilität auftreten, während der Simulant seine Spannung, die eine mehr allgemeine Herabsetzung der Reaktion zur Folge hat, schwerlich aufgeben kann, ohne auch auf dem angeblich analgetischen Gebiete ausgiebige Reaktionen zu liefern.

Die hier erörterte Frage berührt sich mit einer weiteren, die zunächst. nur psychologisches Interesse zu haben scheint, nämlich ob unbewußt bleibende Erregungen in psychogalvanischen Reaktionen Ausdruck finden. Diese Frage hat auch jene Autoren beschäftigt, welche die Ausdrucksphänomene mit den früher üblichen Methoden untersuchten und gewann auch klinische Bedeutung ${ }^{1}$ ). Versuche mit dem Spiegelgalvanometer, die Radecki anstellte und bei denen unterbewußte Prozesse fast stets Reaktionen ergaben, können ihrer ganzen Anlage nach nicht befriedigen; Peterson und Jung fanden deutliche Galvanometerausschläge, die als Komplexreaktionen aufzufassen waren, ohne daß der Komplex ins Bewußtsein der Versuchsperson trat.

Die Auffassung der übergroßen Ausschläge bei Hysterischen als Komplexreaktion führt uns dazu, die Bedeutung des psychogalvanischen Phänomens für die Tatbestandsdiagnostik zu erörtern. Diese Methode hat ihre allgemeingebräuchliche Gestalt im Assoziationsversuch bekommen. Eine Bereicherung der dabei feststellbaren Komplexmerkmale durch das psychogalvanische Experiment, wie es Peterson und Jung, besonders aber Binswanger ${ }^{2}$ ) mit Erfolg versuchten, muß um so willkommener erscheinen, als der Wert des gewöhnlichen Ver-

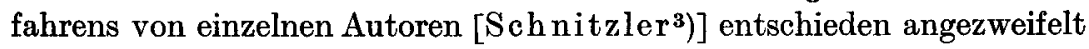
wurde. Ehe ich aber auf diesen Teil der Frage eingehe, möchte ich eire andere Art von Versuchen besprechen, welche an eine ältere Anordnung So $\mathrm{mers}^{4}$ ) über das Gedankenlesen anknüpfen. Auf diese Weise dürfte der Leser vielleicht am schnellsten zu einem Urteil über die Empfindlichkeit unserer Methode, zumal im Vergleich zu anderen Hilfsmitteln gelangen.

Bei diesen Versuchen wurde der Versuchsperson eine Anzahl von Bildern, Worten oder Zahlen gezeigt, aus denen sie eines sich zu merken hatte. Hierauf wurden die Glieder der Gruppe, aus der die Wahl erfolgte, der Reihe nach vorgeführt. Gleichzeitig wurden aber jetzt die psychogalvanischen Schwankungen des Saitengalvanometers photo-

1) Gregor und Zaloziecki, l. c.

2) Binswanger, L., Ửber das Verhalten des psychogalvanischen Phänomens beim Assoziationsexperiment. Journ. f. Psychol. u. Neurol. 10, 149. 11, 65 u. 133. 1908.

3) Schnitzler, J. G., Experimentelle Beiträge zur Tatbestandsdiagnostik. Zeitschr. f. angew. Psychol. 2, 51. 1908.

4) Sommer, R., Dreidimensionale Analyse von Ausdrucksbewegungen. Zeitschr. f. Psychol. 16, 275. 1898. 
graphisch registriert und auch der Zeitpunkt, in dem jede einzelne Karte der Versuchsperson vorgeführt wurde, durch Druck auf einen Taster mittels Federsignal auf der Kurve markiert. Nach Abschluß des Versuches hatte die Versuchsperson das gewählte Objekt zu benennen. Da bei unserer Versuchsanordnung auch Zittern der Hände in der Kurve zum Ausdruck kommt, ist ein Vergleich mit dem von Sommer angegeben Verfahren ermöglicht. Doch stellte ich auch eigene Versuche unter gleichen Bedingungen mit dem Sommerschen Apparate an, weil im psychogalvanischen Experimente die Bedingungen für das Auftreten von Zittern weniger günstig sind.

Der Vergleich beider Versuchsanordnungen fiel entschieden zugunsten der psychogalvanischen Registrierung aus, da diese weitaus überzeugendere Resultate ergab.

Ich habe nun festzustellen, daß derartige Versuche über Wiedererkennen gelingen und daß man aus den dabei gewonnenen Photogrammen das von der Versuchsperson gewählte Objekt erkennen kann. Ferner ist darauf hinzuweisen, daß derartige Versuche ein großes, praktischforensisches Interesse beanspruchen dürfen; man denke nur an den keineswegs seltenen Fall, daß es darauf ankommt, zu ermitteln, ob ein bestimmter Gegenstand (Corpus delicti) zu einem bestimmten Individuum in besonderer Beziehung steht. Daß in diesem Falle die angegebene Versuchsanordnung auch dem Assoziationsexperiment überlegen ist, halte ich für sicher. Meines Ermessens wäre in allen Fällen, in denen Tatbestandsdiagnostik zu forensischen $Z$ wecken versucht wird, in erster Linie daran zu denken, ob die hier beschriebene Methode Anwendung finden kann. Sehen wir von dem Apparatenkomplex ab, so spricht entschieden für sie

1. ihre Voraussetzungslosigkeit, denn ihr zu genügen bedarf es keiner besonderen Bildung oder Intelligenz;

2. geringe Abhängigkeit vom Willen des Individuums; denn sich dem Assoziationsexperimente durch beharrliches Schweigen zu entziehen liegt näher, als sich dem Eindrucke einfacher Reize zu verschließen. Auch ist zu berücksichtigen, daß Komplexmerkmale beim Assoziationsexperiment mitunter ohne Komplex auftreten und der rein sprachliche Mechanismus einen Komplex verdecken kann.

3. Die einfache Deutung der psychogalvanischen Reaktionen bei einer derartigen Versuchsanordnung.

Bei der praktischen Bedeutung, welche derartige Versuche gewinnen, können, möchte ich hier noch besonders auf die Kautelen hinweisen, welche dabei unbedingt zu beachten sind:

1. Die Versuchsperson darf nie unmittelbar an derartige Versuche herantreten, sondern erst durch eine Reihe von Vorversuchen in anderen Sitzungen an die besondere Versuchsanordnung des psycho- 
galvanischen Experimentes gewöhnt werden. Auf diese Weise lernen wir auch ihre Reaktionsart kennen und werden instand gesetzt, eventuelle zufällige Schwankungen oder abnorme Ausschläge zu bewerten.

2. Es muß für größte Ruhe in der Umgebung der Versuchsperson gesorgt sein, zufällige, ungewohnte Eindrücke sind abzuhalten.

3. Die entscheidenden Versuche sind derart anzustellen, daß vor Exposition der Gruppe von Reizen, denen der kritische angehört, der Versuchsperson erst eine Reihe anderer, gleichwertiger vorgeführt werden, da bekanntlich auf die ersten Eindrücke in jedweder Versuchsanordnung relativ starke Ausschläge erfolgen. Die hier geforderte Versuchsbedingung ist dann als erfüllt anzusehen, wenn weder die Ruhekurve gröbere Schwankungen zeigt noch beim Vorzeigen von Objekten, die dem kritischen gleichwertig sind, stärkere Reaktionen erfolgen.

Die anderen Kautelen fallen mit den Versuchsbedingungen beim üblichen diagnostischen Assoziationsexperiment zusammen und brauchen hier nicht eigens erörtert zu werden.

Eine andere Verwendung des psychogalvanischen Phänomens zur Diagnostik des Tatbestandes ist in seiner Verbindung mit dem Assoziationsexperiment gegeben, welches sich ja in dieser Hinsicht bisher vielfach dienlich erwiesen hatte. Veraguth stellte zwei Reihen derartiger Versuche an, bei der einen hatte die Versuchsperson auf das ihr zugerufene Reizwort zu reagieren, bei der anderen nicht. Er erbrachte den Beweis, daß unter beiden Anordnungen psychogalvanische Reaktionen auftreten. Auch zeigten seine Versuche deutliche Komplexreaktionen. Für eine Klärung der Frage sind sie aber zu wenig durchsichtig und leiden unter dem Mangel objektiver Registrierung. Die Stellung des psychogalvanischen Phänomens unter den übrigen Komplexmerkmalen studierten eingehender zuerst Peterson und Jung, die ihre Ergebnisse mit Rücksicht auf die später veröffentlichten Untersuchungen von Binsw a nger nur als vorläufige aufgefaßt wissen wollten. Dieser unternahm ausgedehnte Versuche über die Verwendbarkeit des psychogalvanischen Phänomens für die Tatbestandsdiagnostik. Seine Versuche sind jedoch nach den Anforderungen, die wir heute an psychogalvanische Experimente stellen müssen, ohne Nachprüfung nicht vollständig zu akzeptieren. Zunächst können wir nur mit seinen Ergebnissen bei seiner ersten und einem Teile der Resultate bei der zweiten Versuchsperson rechnen. In der Versuchsreihe c des II. Versuches und in den ganzen Versuchen III und IV befand sich die Versuchsperson in einem besonderen, affektiven Zustande. Nun ergaben aber meine Versuche, daß selbst bei leichter affektiver Erregung die Reaktionen im hohen Maße beeinflußt werden. Da das Vorhandensein einer solchen oft nicht ohne weiteres merklich ist und nur aus den Kurven entschieden werden kann, so haben nur jene Versuche auf unbedingte Anerkennung 
Anspruch, deren einzelne Kurven objektiv aufgenommen wurden. Einen Ersatz hierfür bietet eine eingehende Beschreibung der Versuchsanordnung, sofern aus ihr hervorgeht, daß die Versuchsperson sich in einem indifferenten Gemütszustande befand. Dies ist aber wie erwähnt nur in Versuch I und II a und b der Fall.

Versuche über Tatbestandsdiagnostik unter Benützung des psychogalvanischen Phänomens stellte in jüngster Zeit Moravesik ${ }^{1}$ ) an, in denen er auf Selbstverrat durch Assoziationen und psychogalvanische Komplexreaktionen ausging. Die von ihm benützte Veraguthsche Anordnung ergab aber dabei keine positiven Resultate.

Radecki ließ fortlaufend assoziieren und registrierte dabei die galvanometrische Ablenkung. Um einer eventuell verheimlichten Komplex aufdecken zu können, unternahm er jedesmal zwei derartige Versuchsreihen, indem er die Versuchsperson von 2 verschiedenen Worten ausgehen ließ. Die Deutung setzte bei den Worten ein, bei denen der größte Ausschlag erfolgte. Von 10 derartigen Versuchen ergaben 8 ein positives Resultat.

Da von mir bisher bloß orientierende Versuche vorgenommen wurden, so besteht auch auf diesem Gebiete noch eine Lücke, die durch eine systematische Untersuchung auszufüllen wäre. Methodisch sind hier folgende Fragen von Bedeutung:

1. Laufen die Resultate beider Versuchsanordnungen parallel, d. h. decken sich die Komplexmerkmale des psychogalvanischen und des Assoziationsversuches?

2. Zeigt die psychogalvanische Registrierung Komplexe an, welche beim einfachen Assoziationsexperiment verhüllt bleiben und umgekehrt?

3. Können die beiden Versuchsarten in dieser Verbindung störend aufeinander einwirken?

4. Wie gestalten sich die zeitlichen Verhältnisse der psychogalvanischen Reaktion zumal im Hinblick auf die Größe des Ausschlags?

Binswanger zeigte, daß, worauf auch schon $\mathbf{J} u n g$ und Peterson aufmerksam machten, in vielen Fällen die Reaktionszeit und der Galvanometerausschlag im Assoziationsversuche nebeneinander positive Komplexmerkmale abgeben. Doch war dieser Parallelismus in allen diesen Versuchen kein strenger. Binswanger beobachtete sowohl starke Galvanometerausschläge bei sonst negativen Komplexmerkmalen, aber auch Mangel von Ausschlägen oder eine kleine Galvanometerablenkung in Versuchen, bei denen ein Komp'ex berührt wurde und aus anderen Merkmalen, z. B. der Reaktionszeit erschlossen werden konnte. Derartige Fälle ergaben sich z. B. dann, wenn der Reaktion mit kleinen Ausschlägen aber längerer Assoziationszeit eine Komplexreaktion mit starkem Galvanometerausschlage vorausging. Diese Beobachtung steht

1) l. c. 
im Widerspruch zur Anschauung von J ung und Peterson, daß nach einer ausgeprägten psychogalvanischen Reaktion Neigung zu größeren Ausschlägen besteht.

In anderen Fällen waren die Ausschläge des Galvanometers darum kleiner, als erwartet werden konnte, weil die Versuchsperson infolge eines außerhalb des Versuches gelegenen Erlebnisses (Versuch II c) oder aus spontaner Absicht (Versuch III), endlich infolge der speziellen Versuchsanordnung (Versuch IV) sich in einem besonderen affektiven Zustande befand.

Binswanger faßt diese Erscheinung als eine Hemmung körperlicher Innervationen auf, die infolge äußerer oder innerer Ablenkung zustande kommt. Die Aufmerksamkeit ist dabei nach seiner Ansicht gespalten, anderweitig in Anspruch genommen und alles andere so gehemmt. Ich kann mich dieser Deutung keineswegs anschließen. Zunächst geht die Bezeichnung Hemmung körperlicher Vorgänge von Voraussetzungen über das Wesen des psychogalvanischen Phänomens aus, die bei unseren bisherigen Kenntnissen darüber noch absolut nicht gegeben sind. Ferner faßt Binswanger zu sehr den negativen Effekt ins Auge und berücksichtigt zu wenig, daß in diesen Fällen bei seinen Versuchspersonen ein affektiver Zustand vorlag, der bei unseren sonstigen Erfahrungen über das psychogalvanische Phänomen auch seinerseits in den Versuchen Ausdruck finden mußte. Binswanger geht an dieser Deutung vorbei, indem er die psychische Erregung für den hohen Stand der Kurve z. T. verantwortlich macht. Bei der Erklärung der Erscheinung müßte wohl in erster Linie von dem Kurvenbild ausgegangen werden, und da liegt die Annahme nahe, daß bei einer schon bestehenden Galvanometerablenkung weitere affektive Reize nicht mehr genügend zur Geltung kamen. Für diese Erscheinung habe ich in meinen Kurvenbildern deutliche Belege. Eine weitere Frage betrifft den Bewußtseinszustand des Individuums. Rein psychologisch ist es ohne weiteres als möglich zu bezeichnen, daß bei stärkerer Inanspruchnahme der Versuchsperson durch einen Affekt oder Konzentration auf irgendeinen Vorgang, keine weitere affektive Erregung zur Geltung kommt, also auch keine Komplexe mehr angeregt werden. Aber dies scheint ja gerade in Binswangers Versuchen nicht der Fall gewesen zu sein, da andere Merkmale den Komplex bezeugten.

Wir stehen also vor der Frage, warum fanden in diesem Falle keine entsprechenden psychogalvanischen Reaktionen statt und müssen sie in dem genannten Sinne beantworten.

Bei dieser Divergenz der in beiden Versuchsreihen gefundenen Komplexmerkmale ist an eine mehr methodische Inkongruenz zu denken. $\mathrm{Ob}$ auch eine materielle vorliegt, können erst weitere Versuche entscheischeiden; welche technisch nach Seite der psychogalvanischen Auf- 
nahmen einwandfrei sind. Dazu wäre namentlich erforderlich, daß vor jedem neuen Reize ein auch in der Kurve ersichtlicher indifferenter affektiver Zustand der Versuchsperson vorliegt.

Eine weitere Möglichkeit, daß das psychogalvanische Phänomen durch den Assoziationsversuch gestört werde, hat auch Binswanger erwogen, nämlich ob nicht mehr oder weniger zufällige Störungen im Assoziationsversuche, wie Verhören des Reizwortes zu großen Galvanometerausschlägen führen können. Er nennt einige Fälle, bei denen diese Möglichkeit entschieden nicht eintrat. Die Galvanometerausschläge waren klein, obzwar die Störung zu einer Verlängerung der Reaktionszeit führte. Dabei ist aber zu erwägen, daß die Versuche, die für uns in Betracht kommen, an intelligenten und geübten Versuchspersonen durchgeführt wurden. $\mathrm{Ob}$ auch weniger intelligente, zu Befangenheit neigende Individuen unter diesen Bedingungen affektiv unberührt bleiben, ist wohl noch festzustellen.

Die Kritik der Tatbestandsdiagnostik versäumte es nicht, darauf hinzuweisen, daß durch Sprachgewandtheit Komplexe verhüllt werden können. Es ist darum als Fortschritt in der ganzen Frage zu bezeichnen, $\mathrm{da} ß$ Binswanger auch unter diesen Bedingungen, also trotz kurzer Reaktionszeit durch das psychogalvanische Phänomen auf Komplexe geführt wurde. Die Empfindlichkeit der Methode, d. h. wie weit sie überhaupt Komplexe aufzudecken imstande ist, wäre allerdings noch zu bestimmen. Der Weg dazu liegt in einer noch subtileren Analyse und in der Stiftung besonderer Komplexe, die das Experiment aufzudecken hat.

Eine weitere Möglichkeit. der Störung liegt darin, daß bei weniger sprachgewandten Individuen eine aus rein intellektuellen Momenten verlängerte Assoziationszeit durch affektive Erregung zu Galvanometerausschlägen führt. In diesem Falle würden Komplexe natürlich besonders leicht vorgetäuscht werden.

Die bestehenden oder auch nur möglichen Inkongruenzen zwischen Assoziationsexperiment und psychogalvanischer Aufnahme lassen sich dadurch vermeiden, daß man den psychogalvanischen Versuch vom Assoziationsexperiment ablöst. Die Versuchsanordnung ist dann eine derartige, daß der Versuchsperson Worte vorgelesen oder schriftlich vorgeführt werden. Thre Aufgabe besteht lediglich darin, den Sinn der Worte aufzufassen. Derartige Versuche wurden schon von Veraguth ausgeführt und fielen positiv aus. Versuche, die ich mit dem Saitengalvanometer anstellte, ließen es rätlich erscheinen, diese Richtung weiter zu verfolgen. Die Wahrscheinlichkeit, daß die Versuche an der aktiven Beteiligung der Versuchsperson scheitern, ist jedenfalls geringer als im Assoziationsexperiment, mindestens können wir ihr Verhalten am Saitengalvanometer kontrollieren. Eine Orientierung über die Schärfe der Methode in dieser Hinsicht nahm ich an einer normalen, intelligenten 
Versuchsperson vor, welche die Reize bald unbeachtet vorbeistreichen zu lassen, bald mit Aufmerksamkeit zu verfolgen hatte. Das jederzeitige Verhalten war ohne weiteres aus den Kurven festzustellen. Die Entscheidung über die Brauchbarkeit und Empfindlichkeit beider Verfahren werden erst weitere Untersuchungen treffen können.

Beim Studium der zeitlichen Verhältnisse der psychogaIvanischen Reaktion im Assoziationsversuche sind wir ausschließlich auf die Beobachtungen am Saitengalvanometer angewiesen. Sie haben eine kompliziertere Versuchsanordnung und gebildete Versuchspersonen zur Voraussetzung. Ich stellte sie in der Weise an, daß der Versuchsperson, die wie immer sich in einem abgesonderten Zimmer befand, die Reizworte am Albersschen Apparate vorgeführt wurden. Die sprachliche Reaktion wurde vom Roemerschen Schlüssel aufgenommen, Stromschluß und -Öffnung wurden durch ein Federsignal am Kurvenblatte markiert. Als Zeitmaß diente ein Jaquetsches Chronometer. Die bisher bloß an einer Versuchsperson ausgeführten Versuche zeigten eine auffallende Unabhängigkeit der Latenzzeit der psychogalvanischen Reaktion von der Assoziationszeit und von der Größe und Dauer des galvanometrischen Ausschlages. Dieses Ergebnis, das sich mit weniger genauen Beobachtungen Radeckis deckt, ist von Bedeutung für die Methodik, da es durch weitere Untersuchungen bestätigt, die kompliziertere Versuchsanordnung überflüssig macht.

Die hier gegebene Übersicht unserer Erfahrungen über das psychogalvanische Phänomen hat wegen mangelhafter Voruntersuchungen vielfach nur vorläufige und der Ergänzung bedürftige Ergebnisse vorgebracht. Sie zeigte aber wobl zur Genüge, daß hier ein weites Arbeitsfeld vorliegt und daß wir überall auf Fragen und Probleme stoßen, die aufzugreifen um so anregender ist, als sie sich vielfach mit praktischen Interessen berühren.

Meinem hochverehrten Chef, Herrn Geheimen Rat Paul Flechsig, dem ich die Möglichkeit verdanke, psychogalvanische Untersuchungen an der psychiatrischen Klinik der Universität Leipzig auszuführen, möchte ich auch an dieser Stelle meinen tiefsten Dank aussprechen. 